\title{
Mechanistic Studies on 14 Electron
}

\section{Ruthenacyclobutanes: Degenerate Exchange with Free Ethylene}

\author{
Patricio E. Romero and Warren E. Piers* \\ University of Calgary, 2500 University Drive N.W., Calgary, Alberta, Canada T2N 1N4 \\ wpiers@ucalgary.ca
}

Supporting Information 
Table S1 $\quad{ }^{1} \mathrm{H}$ and ${ }^{13} \mathrm{C}$ NMR Chemical Shift Data (ppm) for Ruthenacyclobutanes (E 1-4) and for Selected Unsubstituted Late Transition Metallacyclobutanes (E 6-11) for comparison to 2 (E5).

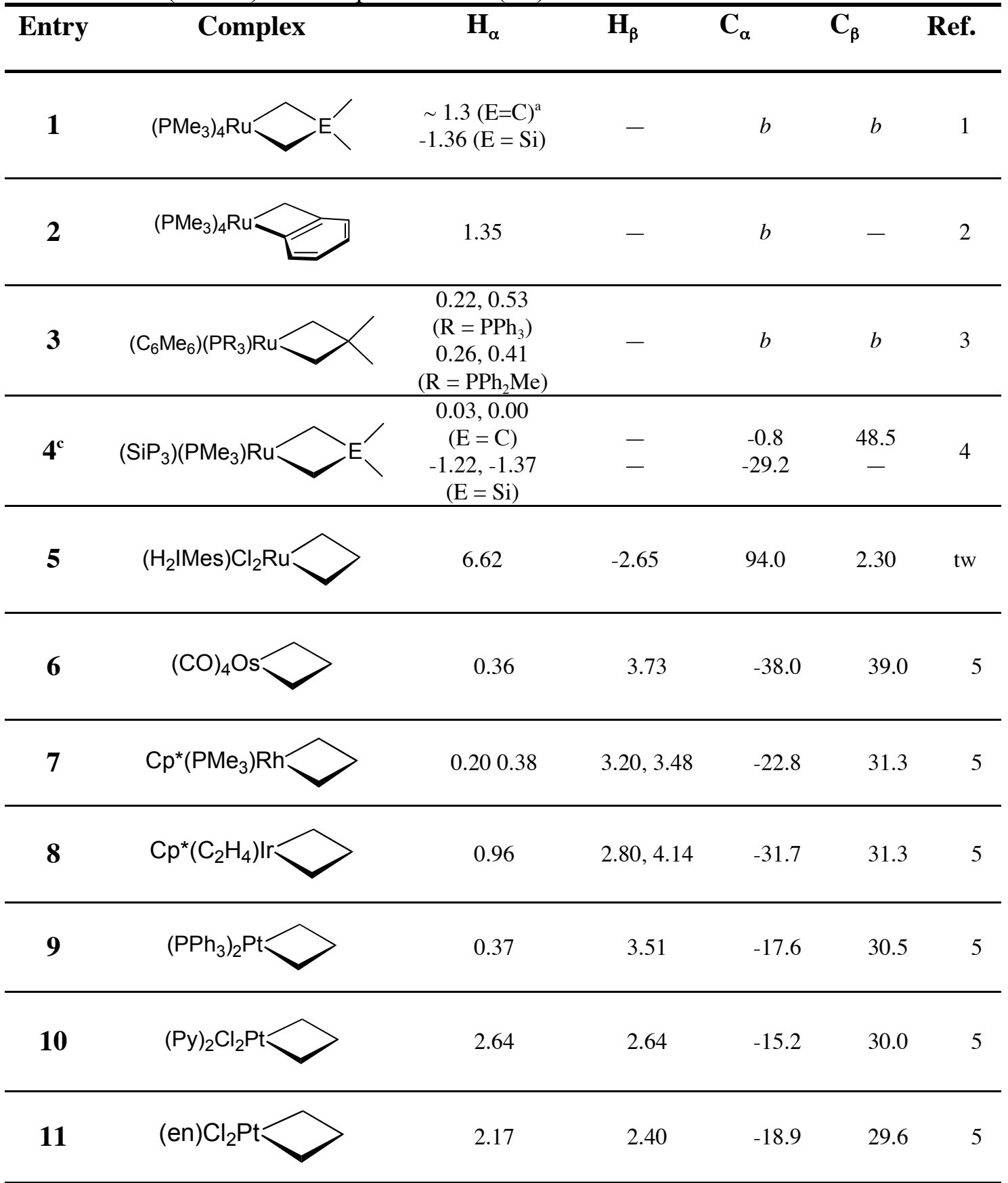

Notes: ${ }^{a}$ Data for the all carbon analogue was only estimated due to multiple overlap of signals. ${ }^{b}$ Not Given. ${ }^{\mathrm{c}} \mathrm{SiP}_{3}=\mathrm{MeSi}\left(\mathrm{CH}_{2} \mathrm{PMe}_{2}\right)_{3}$. ${ }^{\mathrm{d}} \mathrm{en}=$ ethylenediamine. References: 1) Andersen, R. A.; Jones, R. A.; Wilkinson, G. J. Chem. Soc., Dalton Trans. 1978, 446. 2) Statler, J. S.; Wilkinson, G.; Thornton-Pett, M.; Hursthouse, M. B. J. Chem. Soc., Dalton Trans. 1984, 1731. 3) Diversi, P.; Ingrosso, G.; Lucherini, A.; Marchetti, F.; Adovasio, V.; Nardelli, M. J. Chem. Soc., Dalton Trans. 1991, 203. 4) McNeill, K.; Andersen, R. A.; Bergman, R. G. J. Am. Chem. Soc. 1197, 119, 11244. 5) Jennings, P. W.; Johnson, L. L. Chem. Rev. 1994, 94, 2241. tw $=$ this work. 
Table S2 $\quad{ }^{1} \mathrm{H}$ and ${ }^{13} \mathrm{C}$ NMR Chemical Shift Data (ppm) for Selected Early Transition Metallacyclobutanes (E1-9) in comparison to 2 (E10). All data taken from Reference 1; Entryy 10 from this work.

\begin{tabular}{|c|c|c|c|c|c|}
\hline$\overline{\text { Entry }}$ & Complex & $\mathbf{H}_{\alpha}$ & $\mathbf{H}_{\beta}$ & $\overline{C_{\alpha}}$ & $\overline{C_{\beta}}$ \\
\hline 1 & & $b$ & $b$ & 76.7 & -11.6 \\
\hline 2 & & 2.24 & -0.02 & 67.6 & 18.4 \\
\hline 3 & & $\mathrm{~b}$ & $\mathrm{~b}$ & 73.9 & 11.7 \\
\hline 4 & & 2.5 & - & 83.5 & 5.4 \\
\hline 5 & & $\begin{array}{l}3.51 \\
3.11 \\
1.91\end{array}$ & 0.14 & $\begin{array}{c}107.5 \\
76.4\end{array}$ & 20.77 \\
\hline $6^{\mathrm{a}}$ & & 3.86 & 0.48 & 96.1 & -0.68 \\
\hline $7^{\mathrm{a}}$ & A & $\begin{array}{l}4.66 \\
4.51\end{array}$ & $\begin{array}{l}-0.79 \\
-1.39\end{array}$ & 100.7 & -5.10 \\
\hline $8^{\mathrm{a}}$ & & $\begin{array}{l}5.66 \\
4.36 \\
4.04\end{array}$ & -0.77 & $\begin{array}{l}110.8 \\
105.7\end{array}$ & 5.18 \\
\hline $9^{\text {a.c }}$ & & 5.38 & -0.56 & $b$ & $b$ \\
\hline 10 & & 6.62 & -2.65 & 94.0 & 2.30 \\
\hline
\end{tabular}

Notes: ${ }^{a}$ Trigonal Bipyramidal. ${ }^{b}$ Not Given. ${ }^{\mathrm{c}}$ Observed at $-60^{\circ} \mathrm{C}$ in $\mathrm{CD}_{2} \mathrm{Cl}_{2}$. References: 1) Feldman, J.; Schrock, R. R. Prog. Inorg. Chem. 1991, 39, 1. 


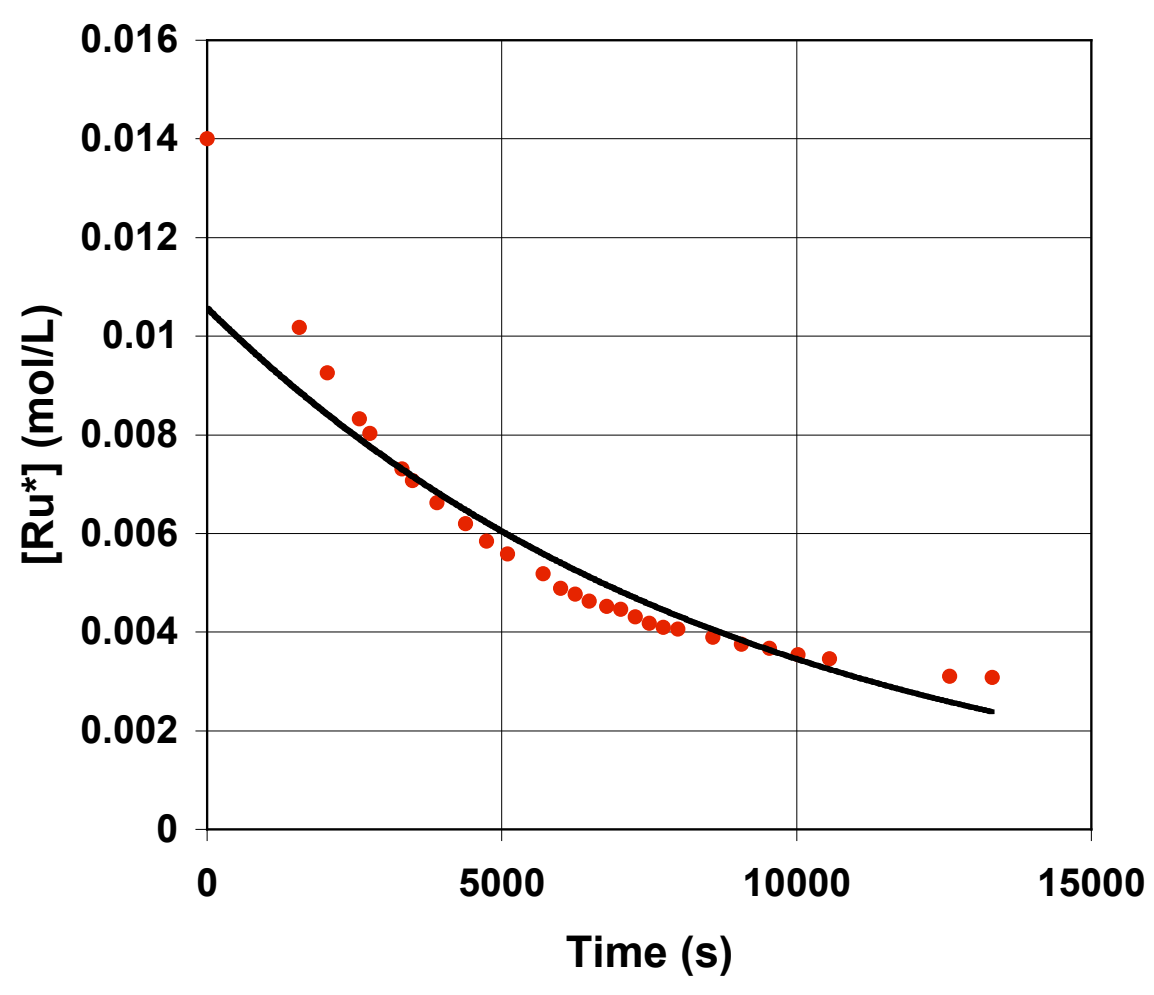

Figure S1 Plot of Conversion of $2-{ }^{13} C_{3}$ into 2 at $-50^{\circ} \mathrm{C}$ using 10 equivalents of $\mathrm{CH}_{2}=\mathrm{CH}_{2}$. Black solid line shows the best exponential fit. 